\title{
Pore-Scale Investigation on the Plugging Behavior of Submicron- Sized Microspheres for Heterogeneous Porous Media with Higher Permeability
}

\author{
Yafei Liu $\mathbb{D},{ }^{1,2}$ Jingwen Yang, ${ }^{1}$ Tianjiang Wu, ${ }^{3}$ Yanhong Zhao, ${ }^{4}$ Desheng Zhou, ${ }^{1,2}$ \\ and Shun Liu ${ }^{1,2}$ \\ ${ }^{1}$ College of Petroleum Engineering, Xi'an Shiyou University, Xi'an, Shaanxi, China \\ ${ }^{2}$ Shaanxi Key Laboratory of Advanced Stimulation Technology for Oil \& Gas Reservoirs, Xi'an, Shaanxi, China \\ ${ }^{3}$ Oil \& Gas Technology Research Institute of Changqing Oil Field Company, PetroChina, Xi'an, Shaanxi, China \\ ${ }^{4}$ Research Institute of Xi'an Changqing Chemical Group Co. Ltd., Xi'an, Shaanxi, China
}

Correspondence should be addressed to Yafei Liu; yafliu@xsyu.edu.cn

Received 12 May 2020; Revised 29 May 2020; Accepted 7 August 2020; Published 17 October 2020

Academic Editor: Andrea Brogi

Copyright $\odot 2020$ Yafei Liu et al. This is an open access article distributed under the Creative Commons Attribution License, which permits unrestricted use, distribution, and reproduction in any medium, provided the original work is properly cited.

\begin{abstract}
Reservoir heterogeneity is regarded as one of the main reasons leading to low oil recovery for both conventional and unconventional reservoirs. High-permeability layers or fractures could result in ineffective water or gas injection and generate nonuniform profile. Polymer microspheres have been widely applied for the conformance control to overcome the bypass of injected fluids and improve the sweep efficiency. For the purpose of examining the plugging performance of submicron-sized microspheres in high-permeability porous media, systematic investigations were implemented incorporating macroscale blocking rate tests using core samples and pore-scale water migration analysis via nuclear magnetic resonance (NMR). Experimental results indicate that microsphere particle size dominates the plugging performance among three studied factors and core permeability has the least influence on the plugging performance. Subsequently, microsphere flooding was conducted to investigate its oil recovery capability. Different oil recovery behaviors were observed for cores with different permeability. For cores with lower permeability, oil recovery increased stepwise with microsphere injection whereas for higher permeability cores oil recovery rapidly increased and reached a plateau. This experimental work provides a better understanding on the plugging behavior of microspheres and could be employed as a reference for screening and optimizing the microsphere flooding process for profile control in heterogeneous reservoirs.
\end{abstract}

\section{Introduction}

Reservoir heterogeneity has long been a major issue that leads to unsatisfactory oil recovery efficiency and high water cut during waterflooding for both conventional and unconventional reservoirs [1-3]. Dominant flow pathways can be developed along high-permeability layers and natural or artificial fractures for tight reservoirs in which injected water or gas prefers to flow hence diminishing the sweep efficiency [36]. Due to the bypass of water, there still remain unswept areas in lower permeability regions with considerable oil recovery potential. In the meantime, high water production could increase the cost and energy consumption of the subse- quent transportation and water treatment. Therefore, it is of significance to adjust waterflooding profile and improve the sweep efficiency to reduce water cut and achieve higher oil recovery efficiency.

To counter the nonuniform waterflood front, profile control technology has been developed to plug the highpermeability layers and divert the flow towards lower permeability areas [4, 7-10]. Methods like polymer flooding [1114] and foam flooding [15-17] were considered to effectively improve the mobility ratio and sweep efficiency. However, issues like shear thinning and injectivity of polymer solution limited its performance in enhancing oil recovery [14]. Oil and water distribution becomes increasingly complex 
TABle 1: Physical properties of the cores used in the experiments.

\begin{tabular}{lccc}
\hline Core no. & Permeability $(\mathrm{mD})$ & Porosity $(\%)$ & Pore volume $\left(\mathrm{cm}^{3}\right)$ \\
\hline 1 & 99 & 30.812 & 7.632 \\
2 & 95 & 31.312 & 7.548 \\
3 & 197 & 20.861 & 4.997 \\
4 & 202 & 20.992 & 5.005 \\
5 & 288 & 28.671 & 7.034 \\
6 & 297 & 27.787 & 6.836 \\
7 & 294 & 28.089 & 6.809 \\
8 & 293 & 26.396 & 6.392 \\
9 & 300 & 27.370 & 6.582 \\
10 & 302 & 28.118 & 6.898 \\
11 & 293 & 28.956 & 6.972 \\
12 & 307 & 29.514 & 7.208 \\
13 & 293 & 29.684 & 6.969 \\
14 & 300 & 29.536 & 6.990 \\
15 & 308 & 28.074 & 6.834 \\
16 & 302 & 27.931 & 6.641 \\
17 & 402 & 25.021 & 6.023 \\
18 & 399 & 28.077 & 6.684 \\
19 & 516 & 26.869 & 6.431 \\
20 & 525 & 27.317 & 6.498 \\
\hline
\end{tabular}

especially during later stage of oil field development such that polymer flooding cannot fully meet the demand for the profile control. Subsequently, polymer particles, for instance, preformed particle gels (PPGs) $[18,19]$, colloidal dispersed gels (CDGs) [20, 21], and polymer microspheres (PMs) [22, 23], were applied to engage in in-depth profile control owing to its small size, elastic characteristics, and the ability to penetrate into lower permeability areas. Extensive studies have been done on the fabrication and performance evaluation on these polymer particles [24]. PPGs are polymers with relatively large size and can be applied for conformance control of fractures and high-permeability layers [25]. The fabrication, mechanical properties, and plugging performance of PPGs have been extensively investigated both in lab and field $[19,26,27]$. CDGs are colloids suspended in the solution with characteristics like smaller size, lower polymer concentration, and longer formation time, yet CDGs should be carefully applied in harsh conditions $[21,28]$. As the complexity of the reservoir pore throat structure raises after water injection and other flooding methods, demand has grown for polymer particles with more flexibility and stability to adjust the injection profile.

Recently, polymer microspheres with nano- to micrometer size were developed and applied for deep profile control. Features like small size, elasticity, and better deformability allow the migration of microspheres towards smaller pores and deeper formation with lower permeability. Different types of polymer microspheres with unique characteristics have been proposed in the literature and widely applied in the oil field [29-33]. The plugging performance of polymer microspheres and the aspects impacting its plugging and migration have been previously investigated [22, 23, 34, 35].
It was proposed that polymer microspheres undergo dynamic plugging in other words plugging, deformation, mobilization, and replugging thus enabling the profile control of deeper formation [30, 31, 36]. Different plugging mechanisms were also directly visualized via transparent micromodel [31] and scanning electron microscopy (SEM) [35]. Majority of the work is focused upon the migration behavior and rheological property of microspheres for deep profile control as well as the compatibility of the particle size to pore throat size. To obtain a solid understanding on the plugging performance of microspheres in a heterogeneous reservoir, systematic investigation on the parameters affecting its plugging performance in both deep formation and high-permeability zones could be essential. However, less attention was paid to the plugging capability of submicron-sized microspheres in higher permeability regions. The controlling factor for microsphere plugging performance remains unclear.

Therefore, in this work, submicron polymer microspheres with varying concentrations and particle sizes have been used to examine its capability to plug highpermeability porous media. To evaluate its plugging performance and investigate the plugging mechanism, a systematic investigation was implemented incorporating the effect of rock permeability, microsphere size, and concentrations on the plugging performance. Macroscale blocking rate of core samples using microspheres as well as the pore-scale inspection of water migration via NMR scanning has been integrated to obtain a better understanding of the plugging mechanism. Based upon the results obtained from this experimental work, influence of each examined parameter on the plugging performance was analyzed and the controlling factor was determined. This work also provides a reference and guidance to optimize the profile control operation in the field application via submicron polymer microspheres in heterogeneous reservoirs.

\section{Experimental Setup}

2.1. Materials. Artificial cores were fabricated using quartz sand and epoxy resin with designated permeability gradient distributed from 100 to $500 \mathrm{mD}$. They were utilized to examine the plugging performance of different microsphere solutions under various permeability conditions and perform coreflooding experiments to evaluate the enhanced oil recovery potential of microsphere solution. The average length and diameter of the cores are $4.9 \mathrm{~cm}$ and $2.5 \mathrm{~cm}$, respectively. Detailed physical properties of the core samples are listed in Table 1. Distilled water and paraffin oil were used to perform the corresponding experiments.

Microspheres used in this work were acrylamide-based polymer particles fabricated via inverse emulsion polymerization. It consists of an outer hydrophilic shell, a middle hydrophobic layer, and a cationic core. Microsphere solution was prepared by diluting the polymer microsphere emulsion with distilled water. Microsphere solutions with varying concentrations $(0.05,0.1,0.15$, and $0.2 \mathrm{wt} \%)$ and particle sizes $(50,100,300$, and $800 \mathrm{~nm})$ were used in the experiments. 


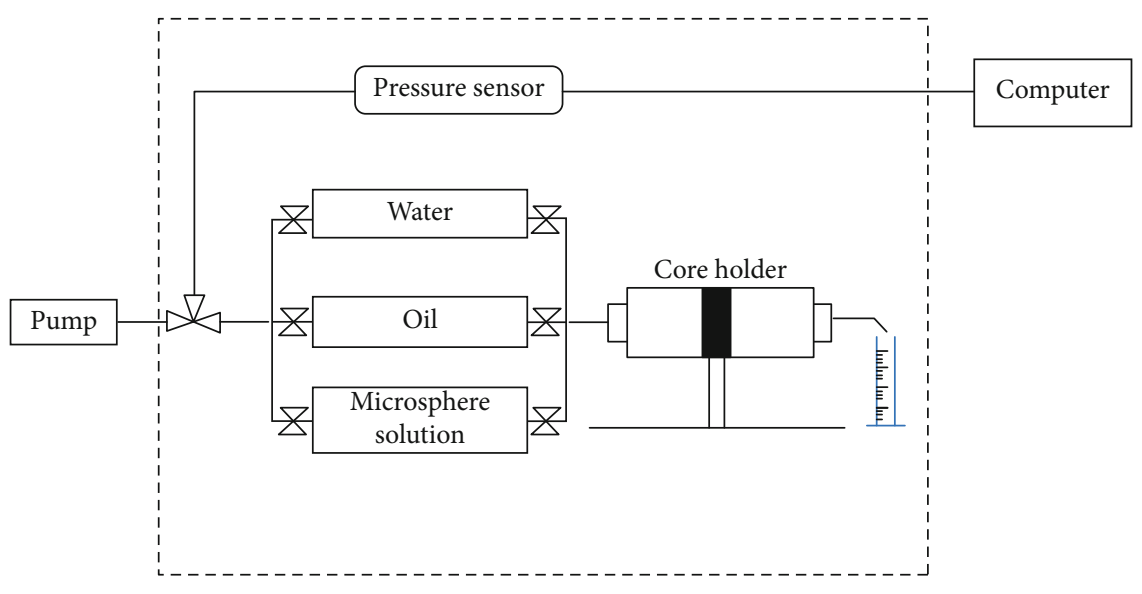

FIGURE 1: Schematic of the experimental setup.

2.2. Experimental Procedures. In order to evaluate the plugging performance of submicron-sized microspheres on the high-permeability regions, microsphere flooding was conducted. The core samples were vacuumed for 3 hours followed by the saturation process with distilled water for 10 hours to ensure that all cores were fully saturated. To obtain the initial distribution of the aqueous phase inside the core, NMR (MesoMR23-60H-I) tests were implemented for all the core samples. Afterwards, the core sample was placed in the core holder. Microsphere solution was then injected into the core with an injection rate of $0.3 \mathrm{~mL} / \mathrm{min}$. Inlet pressure of the core holder was automatically monitored and recorded during the flooding process ranging from 0.01 to $0.6 \mathrm{MPa}$. Volume of the collected displaced fluids was measured to obtain the permeability of the core sample after the microsphere solution injection. The flooding process ceased as the measured pressure became stable. The second NMR test was performed for each core sample afterwards to acquire the information on the aqueous phase distribution and saturation. The whole process was repeated using microsphere solutions with varying concentrations and particle sizes inside differential permeability cores. The experimental setup was sketched in Figure 1.

The aforementioned microsphere flooding experiments were regarded as the preliminary evaluation to screen the optimal microsphere solution concentration and microsphere size and obtain its plugging mechanism. To further examine the oil displacement efficiency by the submicron microspheres, another set of experiments emulating the oil recovery process were conducted. Fresh core samples with two permeability levels were selected based upon the experimental results and evaluation. The core samples experienced the similar preparation steps involving vacuuming and establishment of initial water saturation. Subsequently, initial oil saturation was developed by oil injection at a rate of $0.3 \mathrm{~mL} / \mathrm{min}$ into the core until no more water was produced. Waterflooding and microsphere flooding were conducted afterwards, respectively, at an injection rate of $0.3 \mathrm{~mL} / \mathrm{min}$ until the measured pressure reached a plateau and no more oil was recovered. $10 \mathrm{MPa}$ confining pressure was applied to the displacement system. Recovered oil was collected; therefore, oil recovery rate by two flooding methods was attained.
The experimental workflow for two sets of experiments was also demonstrated in Figure 2.

\section{Results and Discussion}

\subsection{Plugging Performance Evaluation of Microspheres}

3.1.1. Effect of Core Permeability. Core samples with varying permeability were employed to investigate the influence of rock permeability on the plugging performance of microspheres. Two types of microsphere solutions were used for this set of experiments. Core samples used for the experiments were nos. $1,3,5,17$, and 19 for the $100 \mathrm{~nm} 0.1 \%$ case and nos. 2, 4, 6, 18, and 20 for the $300 \mathrm{~nm} 0.1 \%$ case, respectively. Figure 3 plots the relationship between blocking rate and core permeability. Blocking rate was calculated by the ratio of permeability reduction after the microsphere injection to the permeability of the core after water saturation, namely, higher blocking rate implies that larger portion of the pore space is plugged by the injected microspheres. Reversely, lower blocking rate suggests that injected microspheres cannot effectively block larger pores and might pass through the core instead. For the $100 \mathrm{~nm}$ microsphere solution with $0.1 \%$ concentration, the blocking rate fluctuates as the permeability increases yet a distinct trend was not observed and this fluctuation could be possibly due to insufficient particle size that leads to unstable plugging performance, whereas for the $300 \mathrm{~nm}$ microsphere solution with $0.1 \%$ concentration the blocking rate almost remains the same as the permeability increases indicating that microsphere with this particle size demonstrates more stable plugging behavior and better fits the high-permeability cores used in the experiments. In general, this result implies that rock permeability might not be the major factor affecting the plugging capability of the microspheres. When using differently sized microspheres, however, rock permeability could still make a difference in the pore scale. To understand the migration of water after microsphere injection, NMR scanning was conducted after both water saturation and microsphere injection. Pore size distribution of the artificial core samples majorly falls in the range from 0.26 to $7.65 \mu \mathrm{m}$, and they were 


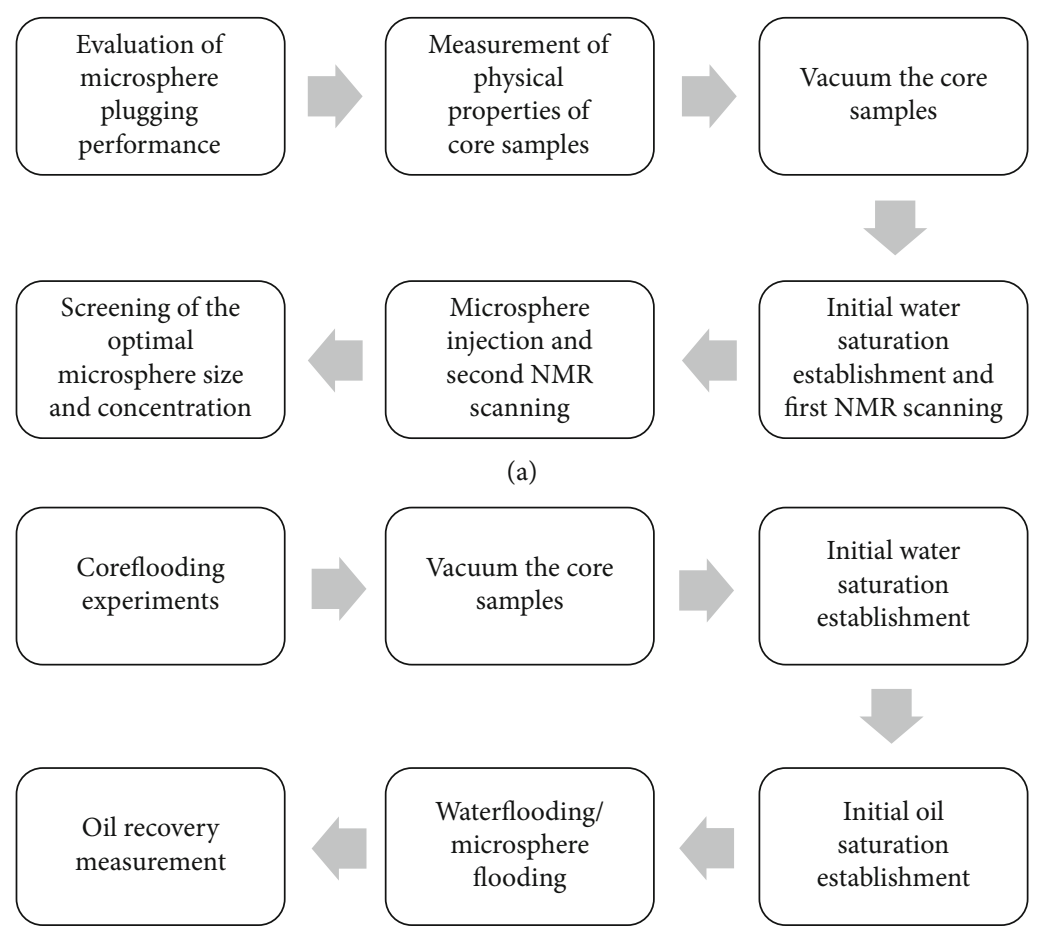

(b)

FiguRE 2: Workflow of the experimental procedures of (a) evaluation on microsphere plugging performance and (b) coreflooding experiments.

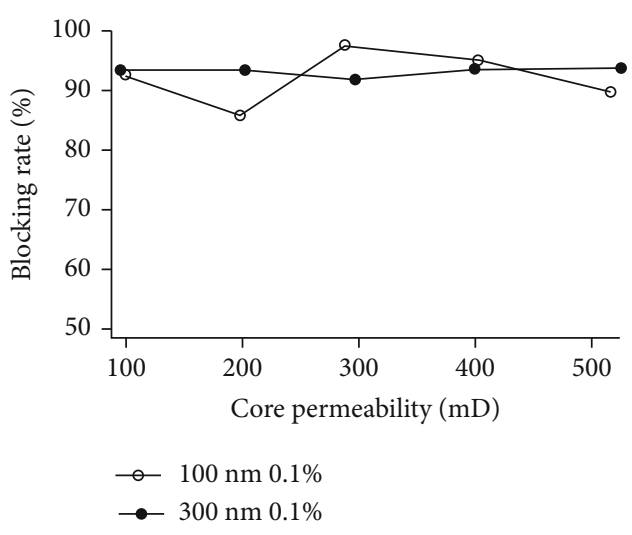

FIGURE 3: Influence of core permeability on the blocking rate of microspheres.

further categorized as small pores, medium pores, and large pores based upon the relaxation time [37].

In Figure 4, water distribution before and after microsphere injection was compared by selecting two core samples with large permeability contrast. Core no. 1 possesses smaller permeability, and NMR scanning shows the majority of the cores characterized as medium pores and large pores are scarce. Water saturation inside medium pores decreased after the microsphere injection, and this portion of water was relocated to small pores suggesting microspheres successfully plugged relatively larger pores and diverted the flow path. For the higher permeability core, majority of the pores are characterized large pores. After microsphere injection, water inside large pores mainly migrated to medium pores and limited amount of water to small pores. Despite the fact that macroscale blocking rate does not change distinctly as rock permeability changes, rock permeability still has an impact on the pore-scale water distribution and relocation as microspheres blocked preferential flow paths.

3.1.2. Effect of Microsphere Size and Concentration. Permeability is the inherent property of the reservoir whereas parameters like microsphere size and concentration are convenient to adjust in the oil field. Therefore, the effect of microsphere size and its concentration was investigated to comprehend the mechanism of profile control using microspheres and optimize the formula for microsphere injection. Figure 5 shows the blocking rate of differently sized microspheres on core samples with the same permeability, and it demonstrated the relationship between blocking rate and microsphere size under two concentration levels. In this set of experiments, core samples with $300 \mathrm{mD}$ permeability were selected. Core samples used for the experiments were nos. 7, 5,6 , and 8 for $0.1 \%$ concentration level and nos. $9,10,11$, and 12 for $0.2 \%$ concentration level, respectively. In general, linear relation was not observed, and in fact, blocking rate shows nonmonotonic change as the microsphere size increases. For the lower microsphere concentration condition, $100 \mathrm{~nm}$ particle size showed better blocking capability possibly attributed to its compatibility with the pore throat size of the core sample. For higher microsphere concentration condition, microsphere with $300 \mathrm{~nm}$ size showed relatively better plugging performance. When microsphere 


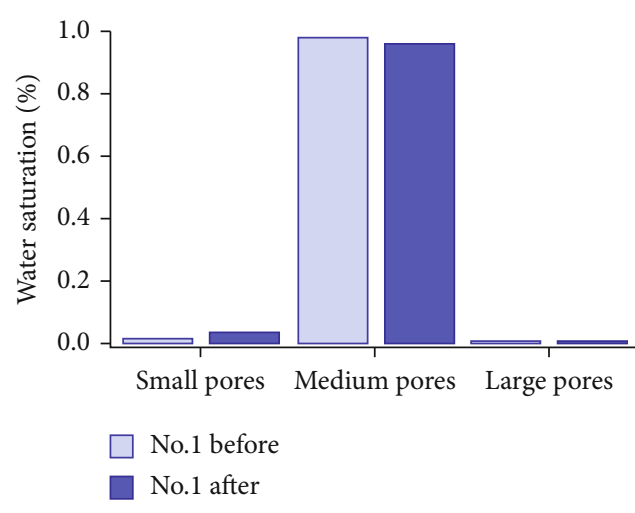

(a)

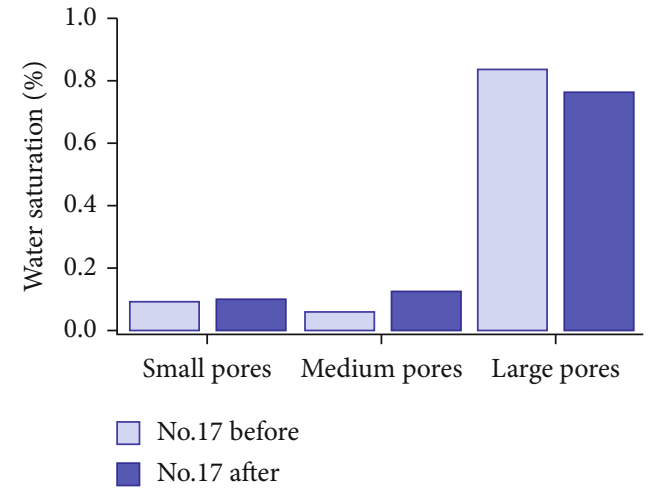

(b)

FIGURE 4: Water distribution inside the core sample with permeability of (a) $99 \mathrm{mD}$ and (b) $402 \mathrm{mD}$. "Before" denotes before microsphere injection and "after" denotes after microsphere injection. Microsphere particle size is $100 \mathrm{~nm}$ and its concentration is $0.1 \%$.

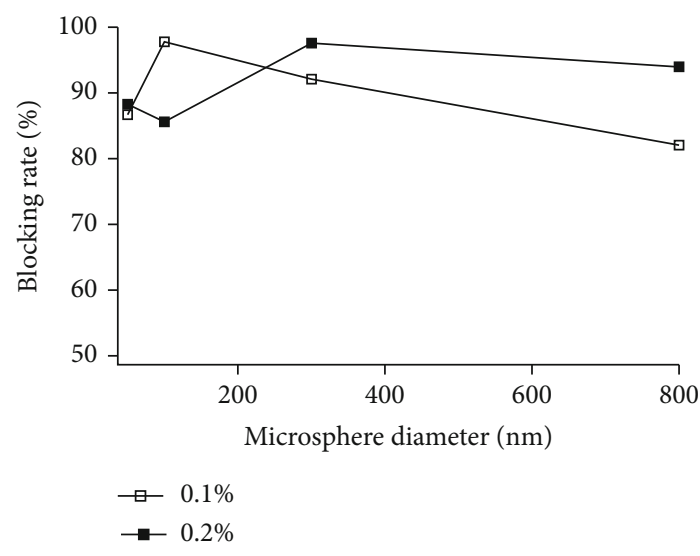

FIGURE 5: Influence of microsphere size on the blocking rate. Permeability of the cores used is $300 \mathrm{mD}$.

solution with larger particle size was used, a discrepancy in blocking rate was observed between two concentration levels. Higher microsphere concentration results in better plugging performance. To analyze the pore-scale migration of water after microsphere flooding with different particle sizes, water distribution change inside two core samples possessing the same permeability was obtained from NMR scanning with the solution concentration of $0.2 \%$. As shown in Figure 6, for core samples with the same permeability, it is intuitive that larger microspheres could effectively block larger pores as indicated by an evident decrease in water saturation in large pores and increase in medium pores after microsphere injection shown in Figure 6(b). Little saturation change in small pores revealed that larger microsphere has a limited effect on plugging small and medium pores despite its nanoto microscale size. Compared with Figure 6(b), Figure 6(a) shows the water saturation change after microsphere injection with smaller particle size using the same permeability core. It is not as effective as larger microspheres in terms of plugging large pores as indicated by less decrease in the water saturation inside large pores.

As for the relationship between blocking rate and microsphere concentration, Figure 7 shows that when larger microspheres were used blocking rate slightly increases as the concentration increases yet the trend flattens out. Further increase of the concentration might not lead to distinct improvement of the blocking rate. Core samples used for the experiments were nos. $13,5,14$, and 10 for $100 \mathrm{~nm}$ particle size and nos. 15, 6, 16, and 11 for $300 \mathrm{~nm}$ particle size, respectively. When smaller microspheres were used, block rate increases as the concentration increases. However, there is an abrupt decrease in the blocking rate at larger concentration especially at $0.2 \%$. It was postulated that for smaller sized microspheres, blocking of larger pores relies upon the accumulation due to its insufficient size. When the concentration was increased to $0.2 \%$, microspheres originally gathered and plugged in the pores could be squeezed out by the subsequently injected microspheres leading to a lower blocking rate, namely, higher microsphere concentration does not necessarily result in higher blocking rate and microsphere particle size should be taken into consideration as well. Presumably, for higher permeability regions, a critical concentration and microsphere size exist and beyond which the growth potential of its plugging performance can be limited. This suggests that for oil field application lower microsphere concentration solution could be more cost-effective considering the expense of the microsphere flooding and elevating microsphere concentration might not lead to considerable improvement in oil recovery. Water saturation change was also plotted in Figure 8 based upon the NMR scanning results. In general, microsphere particles are capable of blocking large pores and divert the fluid flowing towards majorly medium pores and small pores and the blocking of large pores depends upon the specific microsphere concentration and particle size. As shown in Figure 8(b), for microsphere solution with $100 \mathrm{~nm}$ particle size at $0.2 \%$ concentration, the migration of water was not as significant as others and this pore-scale observation coincided with the macroscale blocking rate. Interestingly, core sample no. 15 exhibits different pore size distribution than others that majority of the pore space are characterized as large pores. Microsphere particles still effectively block partial large pores resulting in the migration of water to mainly medium pores and small pores. 


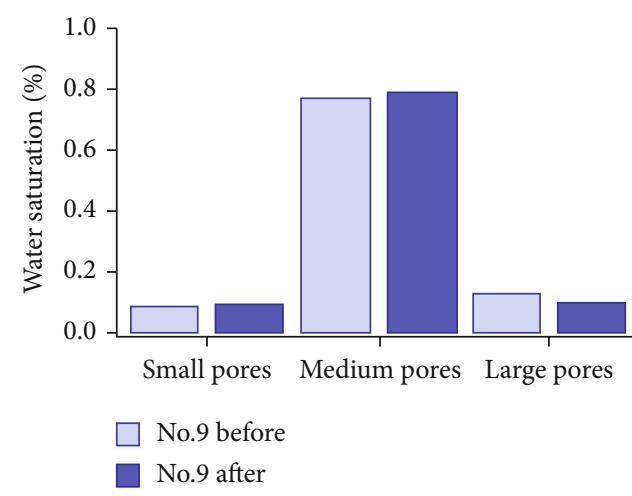

(a)

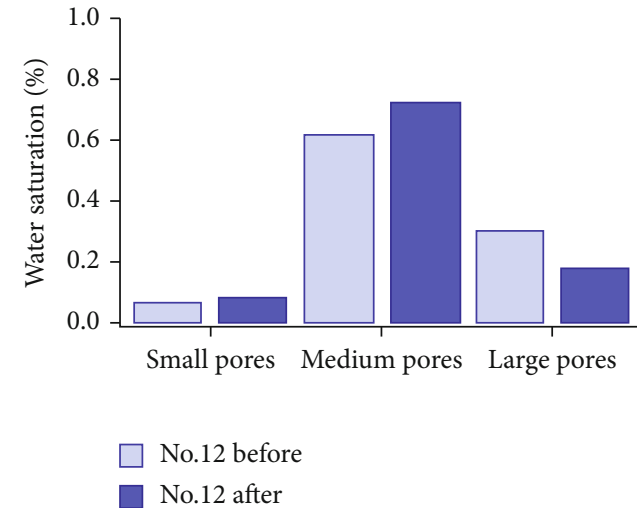

(b)

FIGURE 6: Water distribution inside the core sample of the same permeability ( $300 \mathrm{mD}$ ) after injection of microspheres with (a) $50 \mathrm{~nm}$ and (b) $800 \mathrm{~nm}$ particle size. "Before" denotes before microsphere injection and "after" denotes after microsphere injection. Microsphere concentration is $0.2 \%$.

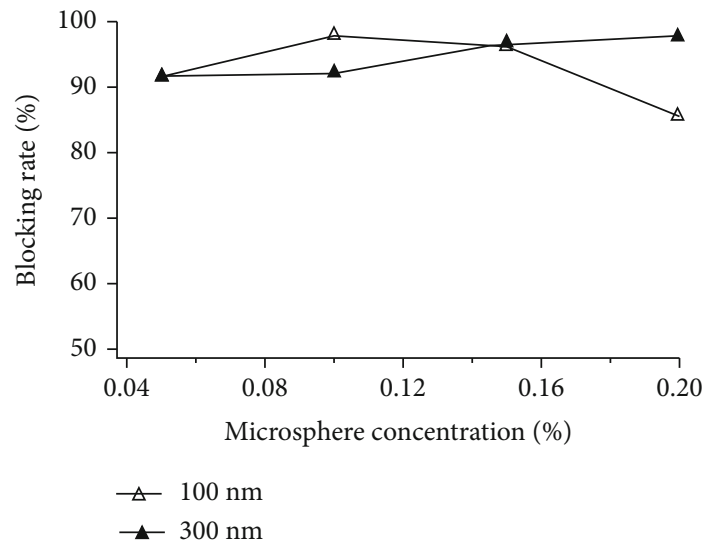

FIGURE 7: Influence of microsphere solution concentration on the blocking rate. Permeability of the cores used is $300 \mathrm{mD}$.

Overall, to determine the dominant factor impacting the blocking rate, statistical analysis was conducted. In this mixed-type orthogonal experiments, three factors were involved which are core permeability, microsphere particle size, and solution concentration. For core permeability, five factor levels were involved and they are 100, 200, 300, 400, and $500 \mathrm{mD}$, respectively. For microsphere size, four factor levels were involved and they are 50, 100, 300, and $800 \mathrm{~nm}$, respectively. For microsphere solution concentration, four factor levels were involved and they are $0.05,0.1,0.15$, and $0.2 \%$, respectively. Based upon the analysis of the range demonstrated in Table 2, the ranking of factors that have greater impact on the blocking performance of microspheres is microsphere size, microsphere solution concentration, and core permeability successively. The corresponding microsphere size and concentration resulting in higher blocking rate were $300 \mathrm{~nm}$ and $0.15 \%$, respectively. Microsphere size was considered as the dominant factor impacting the blocking rate. Therefore, based upon the experimental results, appropriate microsphere size matching with the pore size of the rock could more effectively block the desired region. Core permeability on the other hand has the least influence on the blocking rate among the examined factors indicating submicron-sized microspheres could be applied for profile control in high-permeability regions.

3.2. Oil Displacement Efficiency. To further evaluate the efficiency of enhancing oil recovery by microsphere injection, waterflooding and microsphere flooding were conducted. Four cores with two permeability levels were selected. According to the previous results, microsphere with $300 \mathrm{~nm}$ particle size was chosen to perform the flooding experiments. Details of the experimental setup and results are displayed in Table 3. Generally, incremental oil was recovered by microsphere flooding with the average incremental rate of $9.34 \%$ under the experimental conditions yet the incremental rate depends on the microsphere solution used. The permeability of core no. 10 is three times as large as that of core no. 1; however, the incremental oil recovery rate is similar when the microsphere concentration is $0.15 \%$. On the contrary, when the microsphere concentration decreases to $0.05 \%$, core no. 2 with lower permeability exhibits higher incremental oil recovery rate than core no. 14 . For small-sized microspheres, the blocking capability of large pores possibly depends upon the accumulation of microspheres. Optimal microsphere concentration can be conducive to plug larger pores and ultimately recovery more oil.

Oil recovery rate as a function of pore volumes of microsphere solution injected for the four core samples was plotted in Figure 9 to closely examine the blocking behavior of microspheres. First of all, for lower permeability cores (core no. 1 and no. 2), microsphere concentration has a distinct impact on the oil recovery pattern. Injection of higher concentration solution quickly improves the oil recovery rate and then gradually recovers more oil. The curve also displays step growth of the oil recovery indicating the potential migration of the microspheres into lower permeability areas that leads to the enhanced oil recovery. For core no. 2, it clearly shows a slower response to microsphere injection when the solution concentration is lower. The trend of the curve is 


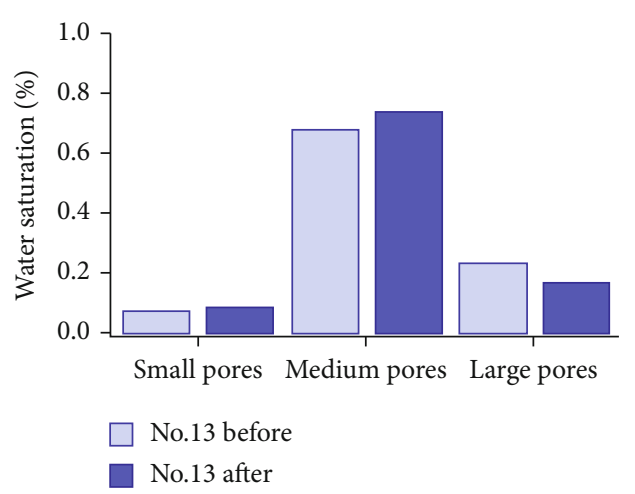

(a)

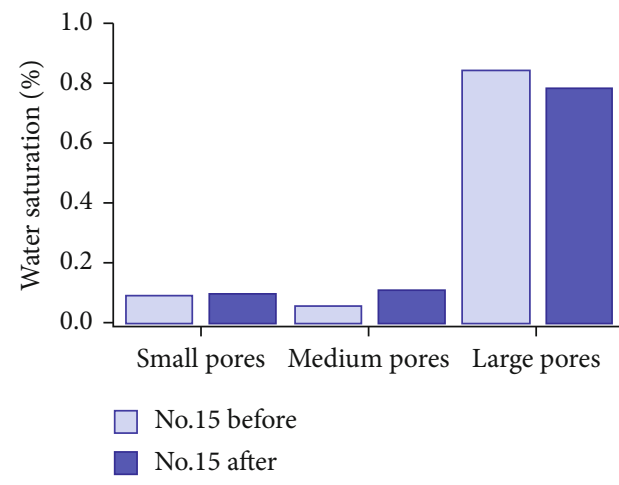

(c)

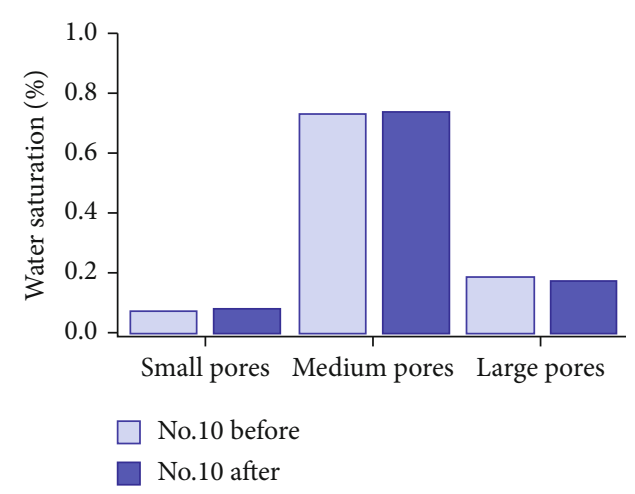

(b)

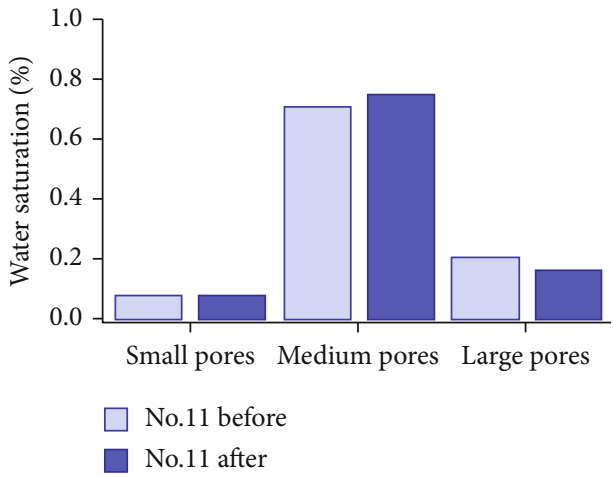

(d)

Figure 8: Water distribution inside the core sample of the same permeability ( $300 \mathrm{mD}$ ) using microsphere solution of (a) $100 \mathrm{~nm} 0.05 \%$, (b)

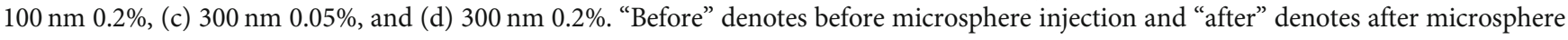
injection.

TABLE 2: Statistical analysis on the factors impacting the blocking rate of microspheres.

\begin{tabular}{lccc}
\hline $\begin{array}{l}\text { Factor } \\
\text { level }\end{array}$ & $\begin{array}{c}\text { Microsphere } \\
\text { size }(\mathrm{nm})\end{array}$ & $\begin{array}{c}\text { Microsphere solution } \\
\text { conc. }(\%)\end{array}$ & $\begin{array}{c}\text { Permeability } \\
(\mathrm{mD})\end{array}$ \\
\hline 1 & 50 & 0.05 & 100 \\
2 & 100 & 0.1 & 200 \\
3 & 300 & 0.15 & 300 \\
4 & 800 & 0.20 & 400 \\
5 & & & 500 \\
Range & 6.17 & 5.09 & 4.77 \\
Order & 1 & 2 & 3 \\
\hline
\end{tabular}

similar to that of core no. 1 demonstrating a step growth characteristic yet the final recovery rate is lower which could be owing to insufficient microspheres in the core and inadequate waterflood profile control. Unlike the lower permeability scenario, when the core permeability is much higher, a rapid increase in oil recovery was observed as the microsphere solution was injected despite its concentration. Presumably, dominant seepage channels are easier to form in higher permeability media, and the gathering of microspheres could effectively divert the flow paths resulting in a significant enhancement in oil recovery. However, the curves for both core nos. 10 and 14 quickly reach a plateau indicating continuous injection of the microsphere solution could
TABLE 3: Oil recovery rate by waterflooding and microsphere solution.

\begin{tabular}{lcccc}
\hline $\begin{array}{l}\text { Core } \\
\text { no. }\end{array}$ & $\begin{array}{c}\text { Microsphere } \\
\text { conc. (\%) }\end{array}$ & $\begin{array}{c}\text { Oil recovery } \\
\text { by } \\
\text { waterflooding } \\
(\%)\end{array}$ & $\begin{array}{c}\text { Oil recovery } \\
\text { by } \\
\text { microsphere } \\
(\%)\end{array}$ & $\begin{array}{c}\text { Incremental } \\
\text { oil recovery } \\
\text { rate }(\%)\end{array}$ \\
\hline 1 & 0.15 & 44.15 & 55.70 & 11.55 \\
2 & 0.05 & 32.41 & 41.00 & 8.59 \\
10 & 0.15 & 53.24 & 64.30 & 12.06 \\
14 & 0.05 & 48.33 & 53.50 & 5.17 \\
\hline
\end{tabular}

sabotage the original plugging by relocating and even flushing out the microspheres such that additional oil recovery was not attained. In this case, increasing solution concentration could be helpful to achieve a higher recovery rate in higher permeability porous media as indicated by comparing the curves of core no. 14 with no. 10 yet the increase might not be significant. In the field application, to plug highpermeability layers using submicron microspheres, an instant improvement could be observed yet the long-term effect of microspheres on oil recovery might not be satisfactory. To further improve the oil recovery, development plan could be adjusted and switched to higher concentration or even larger particle size based on the statistical analysis showing particle size has greater influence on the blocking rate. 


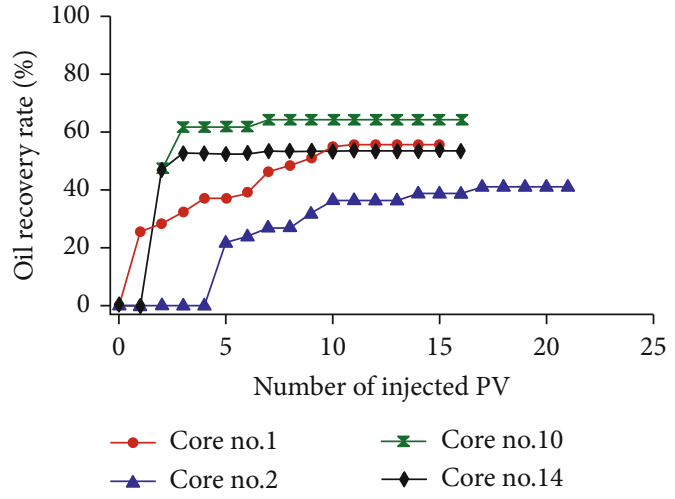

FIGURE 9: Oil recovery rate by microsphere flooding versus number of pore volumes of microsphere solution injected for four core samples.

When a step growth in oil recovery is observed after microsphere injection, increasing its concentration could more effectively enhance oil recovery. To summarize, for a fixed microsphere particle size, higher oil recovery could be achieved by increasing the solution concentration. However, to accomplish higher oil recovery for cores with larger permeability, the effectiveness of increasing solution concentration can be limited.

\section{Conclusions}

In this work, the plugging performance of submicron microsphere solution on high-permeability porous media was investigated. Artificial core samples with designated permeability were employed to examine the plugging capability of different microsphere solutions. Experimental results indicate that microsphere size has a greater impact on the blocking rate than microsphere concentration and core permeability. Larger microsphere mainly blocks large pores whereas smaller microsphere has moderate plugging capability on large pores. The effect of microsphere concentration on the blocking rate should be combined with particle size to evaluate the plugging performance. Noteworthily, core permeability is not the dominant factor impacting the plugging performance based upon the experimental results and statistical analysis. This suggests that small-sized microspheres are capable of blocking higher permeability regions and achieve flow diversion to smaller pores. Simulated oil recovery process demonstrated that incremental oil recovery could be attained by the microsphere injection. Quicker response to the microsphere injection was observed for higher permeability cores. For cores with lower permeability, oil recovery increases stepwise as more microsphere solution is injected until a plateau is reached. When a microsphere solution with the same particle size was used, increasing the concentration could contribute to higher oil recovery yet the increment in oil recovery is associated with rock permeability. Overall, this experimental work could be beneficial for screening the polymer microsphere candidate for profile control of both conventional and unconventional reservoirs that have developed preferential flow paths or fractures to improve the sweep efficiency and ultimately enhance oil recovery.

\section{Data Availability}

The data used to support the findings of this study are available from the corresponding author upon request.

\section{Conflicts of Interest}

The authors declare that they have no conflicts of interest.

\section{Acknowledgments}

This work was supported by the National Natural Science Foundation of China (Grant Nos. 51904244, 51874242, and 51934005), National Major Science and Technology Projects of China (Grant No. 2016ZX05050-009), Natural Science Basic Research Plan in Shaanxi Province of China (Program No. 2019JQ-364), and Scientific Research Program Funded by Shaanxi Provincial Education Department (Program No. 19JK0663).

\section{References}

[1] M. O. Elsharafi and B. Bai, "Effect of weak preformed particle gel on unswept oil zones/areas during conformance control treatments," Industrial and Engineering Chemistry Research, vol. 51, no. 35, pp. 11547-11554, 2012.

[2] P. Wei, W. Pu, L. Sun, Y. Pu, S. Wang, and Z. Fang, "Oil recovery enhancement in low permeable and severe heterogeneous oil reservoirs via gas and foam flooding," Journal of Petroleum Science and Engineering, vol. 163, pp. 340-348, 2018.

[3] G. Zhao, Q. You, J. Tao et al., "Preparation and application of a novel phenolic resin dispersed particle gel for in-depth profile control in low permeability reservoirs," Journal of Petroleum Science and Engineering, vol. 161, pp. 703-714, 2018.

[4] A. M. Almohsin, B. Bai, A. H. Imqam et al., Transport of nanogel through porous media and its resistance to water flow, 2014.

[5] Z. Song, J. Hou, X. Liu, Q. Wei, H. Hao, and L. Zhang, "Conformance control for CO2-EOR in naturally fractured low permeability oil reservoirs," Journal of Petroleum Science and Engineering, vol. 166, pp. 225-234, 2018.

[6] Y. Zhang, M. Gao, Q. You et al., "Smart mobility control agent for enhanced oil recovery during $\mathrm{CO} 2$ flooding in ultra-low permeability reservoirs," Fuel, vol. 241, pp. 442-450, 2019.

[7] S. Vossoughi, "Profile modification using in situ gelation technology-a review," Journal of Petroleum Science and Engineering, vol. 26, no. 1-4, pp. 199-209, 2000.

[8] D. Caili, Y. Qing, and Z. Fulin, "In-depth profile control technologies in China-a review of the state of the art," Petroleum Science and Technology, vol. 28, no. 13, pp. 1307-1315, 2010.

[9] K. S. M. El-Karsani, G. A. Al-Muntasheri, and I. A. Hussein, "Polymer systems for water shutoff and profile modification: a review over the last decade," SPE Journal, vol. 19, no. 1, pp. 135-149, 2014.

[10] B. Bai, J. Zhou, and M. Yin, "A comprehensive review of polyacrylamide polymer gels for conformance control," Petroleum Exploration and Development, vol. 42, no. 4, pp. 525-532, 2015. 
[11] W. B. Gogarty, "Mobility control with polymer solutions," Society of Petroleum Engineers Journal, vol. 7, pp. 161-173, 2013.

[12] H. L. Chang, "Polymer flooding technology yesterday, today, and tomorrow," Journal of Petroleum Technology, vol. 30, pp. 1-113-1-128, 2013.

[13] D. Wang, J. Cheng, J. Wu, and G. Wang, Experiences learned after production of more than 300 million barrels of oil by polymer flooding in Daqing Oil Field, 2002.

[14] K. S. Sorbie, Polymer-improved oil recovery, 2013.

[15] Y. Zhang, X. Yue, J. Dong, and L. Yu, New and effective foam flooding to recover oil in heterogeneous reservoir, 2000.

[16] W. Yan, C. A. Miller, and G. J. Hirasaki, "Foam sweep in fractures for enhanced oil recovery," Colloids and Surfaces A: Physicochemical and Engineering Aspects, vol. 282, pp. 348-359, 2006.

[17] M. A. Fernø, J. Gauteplass, M. Pancharoen et al., "Experimental study of foam generation, sweep efficiency, and flow in a fracture network," SPE Journal, vol. 21, no. 4, pp. 1140-1150, 2016.

[18] A. Goudarzi, H. Zhang, A. Varavei et al., "A laboratory and simulation study of preformed particle gels for water conformance control," Fuel, vol. 140, pp. 502-513, 2015.

[19] A. Farasat, M. V. Sefti, S. Sadeghnejad, and H. R. Saghafi, "Mechanical entrapment analysis of enhanced preformed particle gels (PPGs) in mature reservoirs," Journal of Petroleum Science and Engineering, vol. 157, pp. 441-450, 2017.

[20] H. L. Chang, X. Sui, L. Xiao et al., "Successful field pilot of indepth colloidal dispersion gel (CDG) technology in Daqing Oilfield," SPE Reservoir Evaluation \& Engineering, vol. 9, pp. 664-673, 2013.

[21] M. Bjørsvik, H. Høiland, and A. Skauge, "Formation of colloidal dispersion gels from aqueous polyacrylamide solutions," Colloids and Surfaces A: Physicochemical and Engineering Aspects, vol. 317, no. 1-3, pp. 504-511, 2008.

[22] W. Pu, S. Zhao, S. Wang, B. Wei, C. Yuan, and Y. Li, "Investigation into the migration of polymer microspheres (PMs) in porous media: implications for profile control and oil displacement," Colloids and Surfaces A: Physicochemical and Engineering Aspects, vol. 540, pp. 265-275, 2018.

[23] J. Li, L. Niu, and X. Lu, "Migration characteristics and deep profile control mechanism of polymer microspheres in porous media," Energy Sci Eng, vol. 7, no. 5, pp. 2026-2045, 2019.

[24] M. Abdulbaki, C. Huh, K. Sepehrnoori, M. Delshad, and A. Varavei, "A critical review on use of polymer microgels for conformance control purposes," Journal of Petroleum Science and Engineering, vol. 122, pp. 741-753, 2014.

[25] A. Imqam, Z. Wang, and B. Bai, "The plugging performance of preformed particle gel to water flow through large opening void space conduits," Journal of Petroleum Science and Engineering, vol. 156, pp. 51-61, 2017.

[26] M. A. Lenji, M. Haghshenasfard, M. V. Sefti, M. B. Salehi, and A. Heidari, "Experimental study of swelling and rheological behavior of preformed particle gel used in water shutoff treatment," Journal of Petroleum Science and Engineering, vol. 169, pp. 739-747, 2018.

[27] H. R. Saghafi, "Retention characteristics of enhanced preformed particle gels (PPGs) in porous media: conformance control implications," Journal of Petroleum Science and Engineering, vol. 166, pp. 962-968, 2018.
[28] J. C. Mack and J. E. Smith, In-depth colloidal dispersion gels improve oil recovery efficiency, 1994.

[29] C. Liu, X. Liao, Y. Zhang et al., "Field application of polymer microspheres flooding: a pilot test in offshore heavy oil reservoir," SPE Annual Technical Conference and Exhibition, vol. 6, 2012.

[30] Z. Hua, M. Lin, Z. Dong, M. Li, G. Zhang, and J. Yang, "Study of deep profile control and oil displacement technologies with nanoscale polymer microspheres," Journal of Colloid and Interface Science, vol. 424, pp. 67-74, 2014.

[31] C. Yao, G. Lei, L. M. Cathles, and T. S. Steenhuis, "Pore-scale investigation of micron-size polyacrylamide elastic microspheres (MPEMs) transport and retention in saturated porous media," Environmental Science \& Technology, vol. 48, no. 9, pp. 5329-5335, 2014.

[32] X. Shi and X. Yue, "Migration and plugging mechanisms of self-aggregated microspheres as a novel profile control," Journal of Petroleum Science and Engineering, vol. 184, p. 106458, 2020.

[33] J. Zhang, X. Tan, X. Zhao, Q. He, and Y. Wang, "Experimental study of small-sized polymeric microgel (SPM) in low- or median-permeability reservoirs," Journal of Petroleum Science and Engineering, vol. 190, p. 106829, 2020.

[34] M. Lin, G. Zhang, Z. Hua, Q. Zhao, and F. Sun, "Conformation and plugging properties of crosslinked polymer microspheres for profile control," Colloids and Surfaces A: Physicochemical and Engineering Aspects, vol. 477, pp. 49-54, 2015.

[35] S. Zhao and W. Pu, "Migration and plugging of polymer microspheres (PMs) in porous media for enhanced oil recovery_experimental studies and empirical correlations," Colloids and Surfaces A: Physicochemical and Engineering Aspects, vol. 597, p. 124774, 2020.

[36] H. Yang, L. Hu, C. Chen et al., "Synthesis and plugging behavior of fluorescent polymer microspheres as a kind of conformance control agent in reservoirs," RSC Advances, vol. 8, no. 19, pp. 10478-10488, 2018.

[37] Y. Liu, Y. Shi, L. Liu, X. Yan, D. Zhou, and S. Liu, “Determination of the pore-throat limits for water imbibition in tight sandstone reservoirs through NMR analysis," Journal of Geophysics and Engineering, vol. 16, no. 1, pp. 253-261, 2019. 